\title{
Leukotriene Degradation
}

National Cancer Institute

\section{Source}

National Cancer Institute. Leukotriene Degradation. NCI Thesaurus. Code C40649.

Leukotriene Degradation consists of conjugation, transport, and/or oxidation of a family of oxidative derivatives of arachidonic acid (5-lipoxygenase pathway) (leukotrienes) having potent regulatory effects on the activity of cardiovascular, pulmonary, central neural, gastrointestinal, and immune system cells involved in host defense, immediate hypersensitivity, and inflammation. 